\title{
The Upd3 cytokine couples inflammation to maturation defects
}

Daniela Romão¹, Mariana Muzzopappa ${ }^{1}$, Lara Barrio¹, and Marco Milán ${ }^{1,2,3}{ }^{\text {* }}$

\author{
${ }^{1}$ Institute for Research in Biomedicine (IRB Barcelona), The Barcelona Institute of Science and \\ Technology, Baldiri Reixac, 10, 08028 Barcelona, Spain. \\ ${ }^{2}$ Institució Catalana de Recerca i Estudis Avançats (ICREA)
}

Pg. Lluís Companys 23, 08010 Barcelona, Spain

${ }^{3}$ Lead Contact

*Correspondence: marco.milan@irbbarcelona.org

Phone/Fax: 3493 4034902/34 934037109

Running title: Inflammation and developmental maturation

Keywords: JAK/STAT, cytokines, inflammation, epithelial tumours, hormones

Number of figures: 4 (+ 4 supplementary)

Number of words: 2569 


\section{Summary}

Developmental transitions such as puberty or metamorphosis are tightly controlled by steroid hormones and can be delayed by the appearance of growth abnormalities, developmental tumors or inflammatory disorders, such as inflamed bowel disease or cystic fibrosis (Andersen et al., 2013; Ballinger et al., 2003; Brain and Savage, 1994; Tennessen and Thummel, 2011). Here we used a highly inflammatory epithelial model of malignant transformation in Drosophila (Dekanty et al., 2012; Muzzopappa et al., 2017) to unravel the role of Upd3 - a cytokine with homology to interleukin 6 - and the JAK/STAT signaling pathway in coupling inflammation to a delay in metamorphosis. We present evidence that Upd3 produced by malignant and nearby cell populations signals to the prothoracic gland - an endocrine tissue primarily dedicated to the production of the steroid hormone ecdysone - to activate JAK/STAT and bantam miRNA and to delay metamorphosis. Upd cytokines produced by the tumor site contribute to increasing the systemic levels of Upd3 by amplifying its expression levels in a cell autonomous manner and by inducing Upd3 expression in neighboring tissues in a non-autonomous manner, culminating in a major systemic response to prevent larvae from initiating pupa transition. Our results identify a new regulatory network impacting on ecdysone biosynthesis and provide new insights into the potential role of inflammatory cytokines and the JAK/STAT signaling pathway in coupling inflammation to delays in puberty. 


\section{Highlights}

- Highly inflammatory epithelial tumors cause a strong developmental delay

- Upd3 cytokine produced by malignant and nearby cells signals to the prothoracic gland

- JAK/STAT signal amplifies the systemic levels of Upd3

- JAK/STAT signaling in the ecdysone-producing compartment impacts on the bantam miRNA

\section{eTOC Blurb}

Inflammatory tissues cause developmental delay and animal lethality. Romäo et al present evidence that the Upd3 cytokine is produced by inflammatory and adjacent tissues to cause developmental delay by interfering with the production of the hormone ecdysone. 


\section{Results}

Highly inflammatory tumors produce Dilp8 and Neural Lazarillo to signal to the prothoracic gland

The Drosophila wing primordium, which proliferates exponentially during the larval stage to give rise to the adult wing after metamorphosis, has been extensively used to model epithelial tumorigenesis (PastorPareja and Xu, 2013). RNAi-mediated downregulation of the Spindle Assembly Checkpoint (SAC) gene bub3 in wing primordia leads to high levels of chromosomal instability $(\mathrm{CIN}$, an increased rate in the loss or gain of chromosomes and a hallmark of most solid tumors of epithelial origin) which, upon apoptosis inhibition with the baculovirus protein p35, induces tumor-like overgrowths with an unlimited growth potential [CIN-tumors, (Dekanty et al., 2012; Muzzopappa et al., 2017)]. For simplicity, the combined expression in wing discs of bub3-RNAi and p35 transgenes will be labeled in all figures as CIN. The growth potential of CIN tissues relies on the activation of the JNK pathway to drive the expression of several secreted proteins including the mitogenic molecule Wingless. Larvae containing CIN-tumors entered metamorphosis three and a half days after control larvae (Figure 1A). The presence of an elav-gal80 transgene to block Gal4 activity in the brain (Figure S1A) partially reduced the delay but these larvae still entered metamorphosis two and a half days after control ones (Figure $1 \mathrm{~A})$. The observed developmental delay was accompanied by an increase in larval volume and a non-interrupted feeding behavior (Figure S1B) and was rescued upon feeding with an activated form of the steroid hormone ecdysone (20E, Figure 1B). Blocking JNK also rescued the CIN-induced delay (Figure 1C), and this rescue was not a consequence of reduced tumor growth. Thus, whereas JNK blockage and Wingless depletion reduced tumor growth, the developmental delay was rescued only in the first case (Figure 1C and S1C, D).

Ecdysone biosynthesis in the prothoracic gland (PG) can be positively regulated by two pathways: the developmental pathway, which depends on the release and action of the prothoracic hormone (PTTH) from the brain to activate ERK signaling in the PG (McBrayer et al., 2007), and the nutritional pathway, which depends on insulin (IIS) signaling positively regulating ecdysone production through a double repression mechanism mediated by bantam miRNA (Boulan et al., 2013; Colombani et al., 2005). Dilp8, a relaxin-like protein released from abnormally growing primordia to inhibit entry into metamorphosis by preventing the release of the PTTH hormone (Colombani et al., 2015; Garelli et al., 2015; Vallejo et al., 2015), was 
ectopically induced in CIN-tissues in a JNK-dependent manner (Figure 1D, E and S1E). Consistently, the peak of ERK phosphorylation caused by PTTH release was delayed by $24 \mathrm{~h}$ in larvae containing CINtumors, and Dilp8 depletion restored the ERK peak to its control values (Figure 1F, F'). Surprisingly, targeted depletion of Dilp8 by two different RNAi lines did not rescue the CIN-induced developmental delay (Figure 1H). Consistent with previous reports (Colombani et al., 2012; Garelli et al., 2012), Dilp8 depletion partially rescued the developmental delay of aberrantly growing wing discs and Dilp8 caused a developmental delay when overexpressed with the wing specific $r n$-gal4 driver in otherwise healthy wing discs (Figure S1F, G). We observed that IIS signaling [monitored by a PH-GFP fusion protein used as an indicator of PI3K activity, (Britton et al., 2002)] was downregulated and bantam activity [monitored by a sensor that ubiquitously expresses GFP, carries two perfect bantam fixation sites in its $3^{\prime} \mathrm{UTR}$ and is therefore repressed in the presence of the miRNA, (Brennecke et al., 2003)] was increased in the PG of CIN-larvae, and that these two changes were restored to control levels upon depletion of JNK activity at the tumor site (Figure 1G, G'). Initially described as a target of JNK signaling in Drosophila (Hull-Thompson et al., 2009), the lipocalin Neural Lazarillo (NLaz) was upregulated in CIN-tumors by JNK activity (Figure 1E and S1I). Consistent with the role of NLaz in antagonizing IIS signaling in peripheral tissues (Pasco and Léopold, 2012), ectopic expression of this lipocalin in the PG (with the P0206-gal4 driver) caused a reduction in IIS signaling, an increase in bantam activity and a developmental delay (Figure 1G, G', K). Overexpression of NLaz in wing discs (with rn-ga/4) caused a similar effect on developmental timing (Figure 1J). Although RNAi-mediated depletion of NLaz from the initial tumor site restored IIS signaling levels in the PG, the developmental delay was not rescued and bantam activity levels remained high (Figure 1G, G', I, see also Figure S1J). These results indicate that CIN-tumors impact on the activity of the developmental and nutritional pathways in the PG through Dilp8 and NLaz, and that bantam activity levels in the PG are independently regulated by another JNK-target. We did not detect any major change in IIS signaling or bantam activity in the PG of larvae containing aberrantly growing wing discs (Figure $\mathrm{S} 1 \mathrm{H}$ ), suggesting that the impact on the nutritional pathway is specific to highly inflammatory conditions. 
Upd3 produced by tumor cells regulates bantam activity in the prothoracic gland

We found that the cytokines Upd1 and Upd3 - which bind to the receptor Domeless [Dome, (Brown et al., 2001)] to activate the JAK-STAT pathway - were induced in CIN-tumors (Fig. 2A-C), and that the JAK/STAT pathway was ectopically activated in the PG of larvae carrying CIN-tumors (Fig. 2D, D'). JNK blockage in CIN-tumors reduced Upd1 and Upd3 expression levels in tumor cells and JAK/STAT signaling in the PG (Fig. 2A-D'). These results point to a potential role of JNK-driven expression of Upds in the CIN-induced delay and open the possibility that this delay is a result of JAK/STAT signaling in the PG. Consistent with this proposal, targeted expression of Upd1, Upd3 or activation of JAK/STAT in the PG caused a developmental delay (Fig. 2E and S2B). Interestingly, bantam was activated in the PG under these circumstances (Figure 2F, F'). In contrast, IIS signaling was not affected (Figure 2F, F'). These results indicate that the regulation of IIS signaling in the PG is a consequence of NLaz activity downstream of JNK and independent of JAK/STAT signaling. We noticed that targeted overexpression of PTTH or Dilp8 in the PG did not have any impact on JAK/STAT activity levels (Figure S2B, C). We next addressed the capacity of Upd1 and Upd3 when overexpressed in the wing disc to act as systemic signals that regulate developmental timing. Whereas Upd3 caused a developmental delay when overexpressed in otherwise healthy wing discs and this delay was accompanied by the activation of JAK/STAT signaling and bantam in the PG (Figure 2G, H, H'), overexpression of Upd1 in wing discs did not have any impact on developmental timing (Figure 2G). These results indicate that Upd3, when produced by wing imaginal cells, can act as a systemic signal to induce a developmental delay.

CIN-induced developmental delay relies on Upd3 produced by tumor cells and nearby cell populations

Surprisingly, depletion of upd3 specifically in CIN-tumors did not rescue the observed developmental delay (Figure 3A) and co-depletion of upd1 and upd3 was required to achieve successful rescue of developmental timing (Figure 3B and S3J). Overexpression of Dome ${ }^{\Delta \mathrm{CYT}}$ in tumors, a truncated form of Dome lacking the intracellular domain [Dome-DN, (Brown et al., 2001)], also rescued the CIN-induced developmental delay (Figure 3C) and the JAK/STAT and bantam activity levels in the PG (Figure 3E, G). This rescue was not the simple result of reducing the activity of the JAK-STAT pathway in CIN tissues, as depletion of JAK/STAT 
with RNAi lines against dome or hop did not have any effect on the observed developmental delay (Figure 3D). We support the proposal that the capacity of Dome-DN to rescue the CIN-induced developmental delay relies mainly on its ability to trap Upd ligands. Consistent with this, overexpression of Dome-DN rescued the developmental delay caused by Upd3 overexpression in otherwise wild-type wing disc cells (Figure 2F) and the local and systemic activation of the JAK/STAT signaling pathway caused by CIN-tumors (Figure S4D, see also Figure 3E). Thus, JAK/STAT signaling was induced in several tissues of CIN-larvae including the brain and larval muscles, and this expression was rescued by targeted overexpression of Dome-DN in CIN-tumors. All these results suggest that CIN-tumors produce Upd1 and Upd3 to induce a developmental delay, but only Upd3 is able to act as a systemic signal to activate JAK/STAT signaling and bantam miRNA in the ecdysone-producing compartment.

These results can be explained by two possible scenarios. In the first scenario, Upd1, together with Upd3, might act locally in the wing disc to induce the expression of other long-range systemic signals to regulate, together with Upd3, ecdysone production. Consistent with this notion, overexpression of Dome-DN in CIN tissues reduced the expression levels of Dilp8 and NLaz (Figure S3A, B), and rescued the impact on the activity levels of the IIS signaling pathway at the PG (Figure 3F). However, co-depletion of two of the three systemic signals from the tumor site did not rescue, even partially, the developmental delay (Figure S3C). We noticed that overexpression of Dome-DN in CIN tissues also reduced the expression levels of Upd1 and Upd3 (Figure $3 \mathrm{H}, \mathrm{I}$ ), opening the possibility that a feed-forward loop mediated by the local activities of Upd1 and Upd3 might expand their expression domains to nearby tissues. Consistent with this second scenario, Upd ligands and JAK/STAT signaling were ectopically induced in myoblasts (a nearby nonepithelial population of precursors of adult muscles, Figure 3J) and in larval muscles (Figure 3K, S3D), and this ectopic induction was blocked by targeted overexpression of Dome-DN or targeted co-depletion of upd1 and upd3 to CIN-tumors (Figure 3K, S3D). We also observed the recruitment of a considerable number of hemocytes (a physiological source of Upd3) to the CIN-tumor (Figure S3E). Interestingly, targeted overexpression of Upd3 in myoblasts, larval muscles or hemocytes induced a developmental delay, thereby validating their potential role as a source of Upd3 and their contribution to the $\mathrm{CIN}$-induced delay (Figure S3F-I). 
Five independent experimental observations support the notion that the systemic activity of Upd3, produced by different tissues, contributes to the $\mathrm{CIN}$-induced developmental delay by acting on the PG to activate bantam and block ecdysone production. First, a deletion allele of upd3 ( $\Delta u p d 3$, (Osman et al., 2012)) rescued the $\mathrm{CIN}$-induced developmental delay and restored the activity of the nutritional pathway to control levels in the PG (Figure 4A, B, B'). By contrast, simultaneous depletion of upd3 in CIN-tumors, myoblast progenitors and muscle cells (Figure S4A), or in CIN-tumors and hemocytes (Figure S4B, C) did not rescue the CIN-induced developmental delay, suggesting that more than two different tissues act as Upd3 sources in CIN-larvae. Second, combining the GAL4/UAS and LexA/LexAop systems to drive CIN in larval wing primordia and deplete stat in the PG rescued the CIN-induced developmental delay and the activity levels of STAT in the PG but not in the wing disc (Figure 4C, D, D'). bantam activity levels in the PG were also rescued under these circumstances (Figure 4D, D'). Third, simultaneous depletion of dome in CIN-tumors and PG cells reduced the CIN-induced developmental delay, whereas single depletion of dome in CINtumors did not (Figure 4F, see also Figure 3D and S4D, E). Fourth, simultaneous depletion of bantam activity, with the use of a bantam inhibitor (bantam sponge), in CIN-tumors and PG cells rescued the CINinduced developmental delay, whereas single depletion of bantam activity in CIN-tumors did not (Figure 4G, see also Figure S4D, E). Fifth, LexA/LexAop-mediated depletion of stat in the PG rescued the developmental delay caused by GAL/UAS-driven expression of Upd3 in larval wing primordia (Figure 4E).

\section{Discussion}

The developmental regulation of puberty in humans and metamorphosis in flies relies on the timely production of steroid hormones (Sisk and Foster, 2004; Tennessen and Thummel, 2011). Here we present evidence that flies carrying highly inflammatory tissues present a very strong delay in developmental maturation, similar to human patients suffering from inflammatory bowel diseases such as Crohn's disease and ulcerative colitis, and we identify fundamental roles of the Upd3 cytokine and the JAK/STAT signaling pathway in triggering this delay. In this work, we used an epithelial model of CIN (Clemente-Ruiz et al., 2016; Dekanty et al., 2012; Muzzopappa et al., 2017), to first show that highly inflammatory epithelial tumors cause a developmental delay that is independent of the size of the tumor. We identify the relaxin Dilp8, the lipocalin Neural Lazarillo (NLaz) and the cytokine Upd3 as three secreted proteins regulated by JNK at the 
tumor site that have a strong capacity to act systemically and block from a distance ecdysone production in the PG (Figure 4H). Whereas Dilp8 is known to reduce ecdysone production indirectly by impinging negatively on the production of the PTTH neuropeptide (Colombani et al., 2012; Garelli et al., 2012), our data indicate that NLaz and Upd3 signal to the PG to activate, through the IIS and JAK/STAT signaling pathways, bantam miRNA, a repressor of ecdysone production (Boulan et al., 2013). Despite the expression of Dilp8, NLaz and Upd3 in CIN tissues and their strong capacity to block ecdysone production in the prothoracic gland when overexpressed in epithelial tissues, our experimental data indicate that the initial trigger of the $\mathrm{CIN}$-induced developmental delay is the JNK-driven expression of the systemic cytokine Upd3 and the locally acting cytokine Upd1, and that local and systemic activities of the JAK/STAT pathway play fundamental roles in amplifying the overall levels of Upd3 to induce a robust developmental delay. Whereas autocrine JAK/STAT signaling in the tumor site contributes to amplifying the expression levels of Upd1 and Upd3 at the tumor site, paracrine JAK/STAT signaling mediated by Upd1 and Upd3 contributes to induce ectopic sources of these two ligands in nearby cell populations. Non-autonomous induction of Upd3 expression in myoblasts and larval muscles, recruitment of hemocytes (a natural source of Upd3 expression) and the most probable amplification of these hemocytes as a consequence of high levels of Upd cytokines in the medium would all contribute to amplifying the systemic levels of Upd3. Whether IL-6, the human homologue of Upd3, and the JAK/STAT pathway play a conserved role in puberty delays caused by inflammation is an interesting question that remains to be elucidated. Interestingly, we noticed that systemic depletion of Dilp8 caused a partial rescue of the CIN-induced developmental delay (Figure S4F) whereas targeted depletion of Dilp8 to the tumor site did not (Figure 1H). Based on the impact of JAK/STAT signaling on Dilp8 and NLaz expression (Figure S3A, B), these observations open the possibility that paracrine JAK/STAT signaling might also contribute to amplifying the systemic levels of these two signals to trigger, together with Upd3, a robust developmental delay.

\section{Acknowledgments}

We thank E. Bach, J. Castelli, S. Cohen, M. Dominguez, B. Edgar, D. Harrison, H. Jasper, H. Jiang, B. Lemaitre, P. Leopold, F. Martín, H. Stocker, G. Struhl, J. P. Vincent, the Bloomington Drosophila Stock 
Center (USA), the Vienna Drosophila Resource Center (Austria), and the Developmental Studies Hybridoma Bank (USA) for flies and reagents, and the IRB Advanced Digital Microscopy Facility for technical help. This work was funded by the BFU2016-77587-P and PID2019-110082GB-I00 grants from MINECO (Government of Spain), and ERDF “Una manera de hacer Europa”. We gratefully acknowledge institutional funding from the Spanish Ministry of Economy, Industry and Competitiveness (MINECO) through the Centres of Excellence Severo Ochoa Award, and from the CERCA Programme of the Catalan Government.

\section{Author Contributions}

D.R. and M.Mi. conceived and designed the experiments. D.R., L.B. and M.Mu. performed the experiments. All authors analyzed the data. M.Mi., with the help of D.R., wrote the paper.

\section{Declaration of interests}

The authors declare no competing interests. 


\section{Figures}
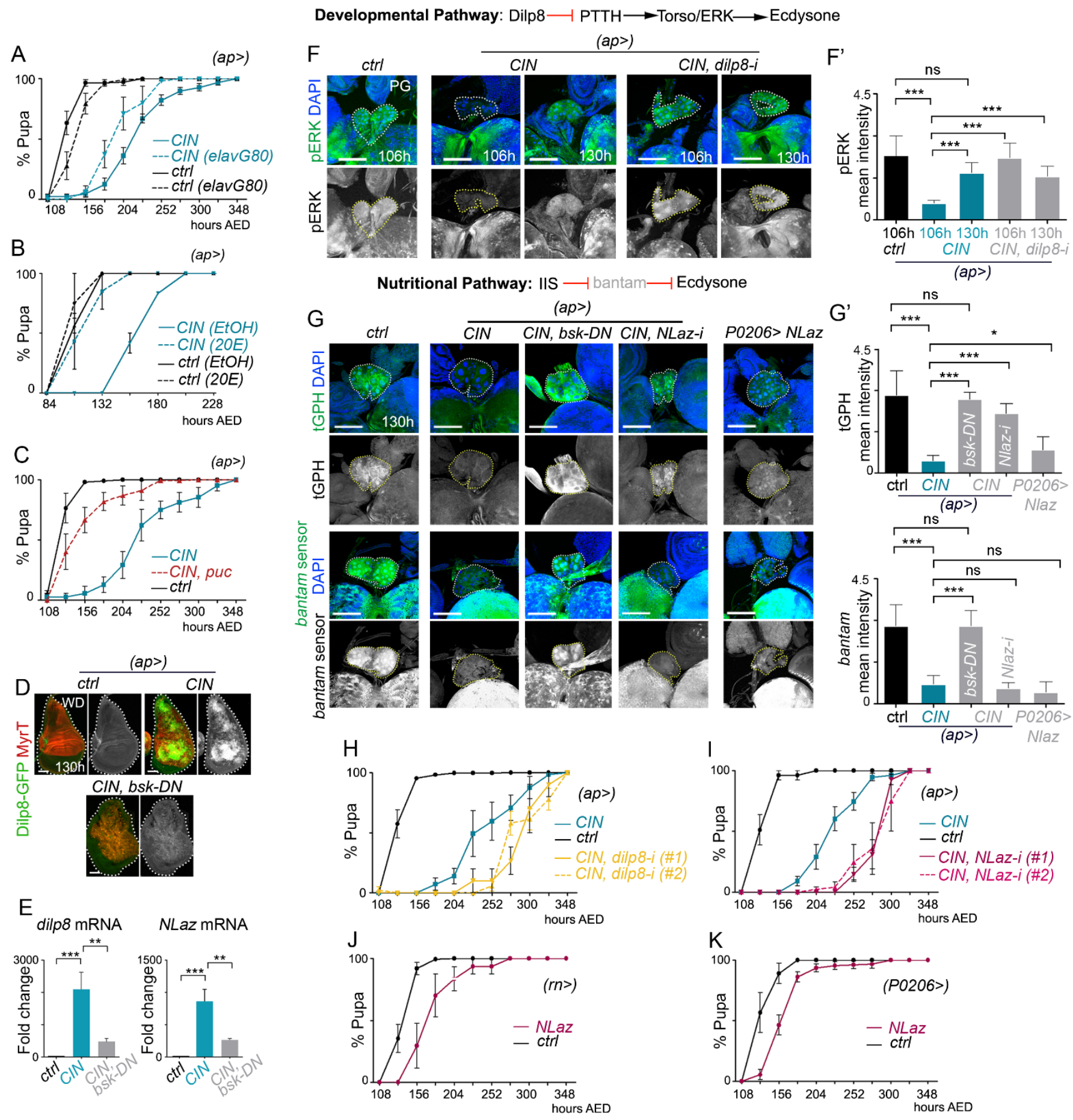

Figure 1. CIN tissues induce a JNK-dependent developmental delay.

(A) Developmental timing of larvae carrying wing discs subjected to CIN with the ap-gal4 driver. (B, C) 20E treatment (B) or puc expression (C) rescued the developmental delay of CIN larvae. (D) Wing imaginal 
discs expressing the indicated transgenes and stained for MyrT (red) and Dilp8-GFP (green). (E) dilp8 and NLaz mRNA levels by qRT-PCR in wing discs expressing the indicated transgenes. $(\mathbf{F}, \mathbf{G})$ Schematic representation of the ecdysone production regulatory networks and ring glands of larvae expressing the indicated transgenes in wing discs (ap-gal4) or in the PG (P0206-gal4), and stained for pERK (green or white, F), tGPH (green or white, G), bantam sensor (green or white, G) and DAPI (blue). (F', G') Mean signal intensity of pERK, tGPH and bantam in PGs of larvae expressing the indicated transgenes in wing discs or PGs. (H-K) Developmental timing of larvae expressing the indicated transgenes in wing discs $(\mathbf{H}$, I, ap-gal4; J, rn-gal4) or in the PG (K, P0206-gal4). Contour of wing discs and ring glands is marked by a dotted line. Scale bars, $50 \mu \mathrm{m}(\mathbf{D}), 100 \mu \mathrm{m}(\mathbf{F}, \mathbf{G})$. Data represent mean \pm SEM (puparation assays and qRT-PCR) or SD (larval volume and mean intensity). ${ }^{* *} p<0.001$, ${ }^{* *} p<0.01$, ${ }^{*} p<0.05$; ns, non-significant. $\mathrm{n}=10-272$ (puparation assays), n=6-14 (mean intensity). AED, after egg deposition. See also Figure S1. 

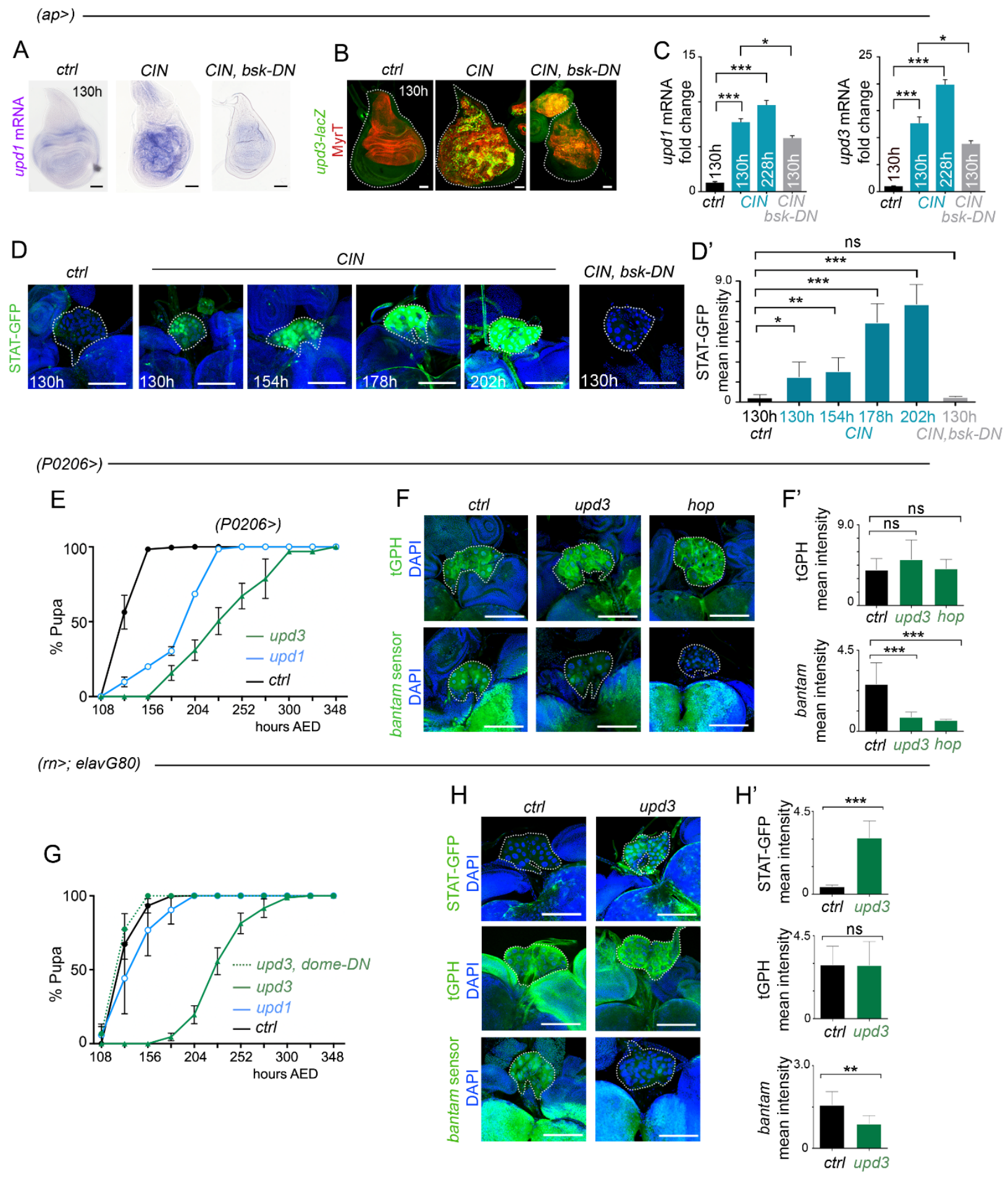

Figure 2. Upd3 produced by tumor cells regulates bantam activity in the prothoracic gland.

(A-B) Wing imaginal discs expressing the indicated transgenes (A, B, ap-ga/4) and stained for upd1 mRNA (purple, A), upd3-lacZ (green, B) and MyrT (red, B). (C) upd1 and upd3 mRNA levels by qRT-PCR in wing discs expressing the indicated transgenes. ${ }^{* *} \mathrm{p}<0.001$, ${ }^{*} \mathrm{p}<0.05$, data represent mean \pm SEM. (D, F, H) 
Ring glands of larvae expressing the indicated transgenes in wing discs (ap-gal4, D, or $r n-g a / 4$, $\mathbf{H}$ ) or in the PG (P0206-gal4, F), and stained for DAPI (blue), STAT-GFP (green, D, H), and tGPH or bantam sensor (green, F, H). Note in G-H' the presence of an elav-gal80 transgene to block Gal4 activity in the brain. (D', F', H') Mean signal intensity of STAT-GFP, tGPH and bantam in PGs of larvae expressing the indicated transgenes in wing discs or PGs. (E, G) Developmental timing of larvae expressing the indicated transgenes in the PG (E, P0206-gal4) or in wing discs (G,rn-gal4), data represent mean \pm SEM. Contour of wing discs and ring glands is marked by a dotted line. Scale bars, $50 \mu \mathrm{m}$ (A, B), $100 \mu \mathrm{m}$ (D, F, H). Data represent mean \pm SEM (puparation assays and qRT-PCR) or SD (mean intensity). ${ }^{* * *} p<0.001,{ }^{* *} p<0.01,{ }^{*} p<0.05$, ns, non-significant. n=38-282 (puparation assays), n=5-15 (mean intensity). AED, after egg deposition. See also Figure S2. 

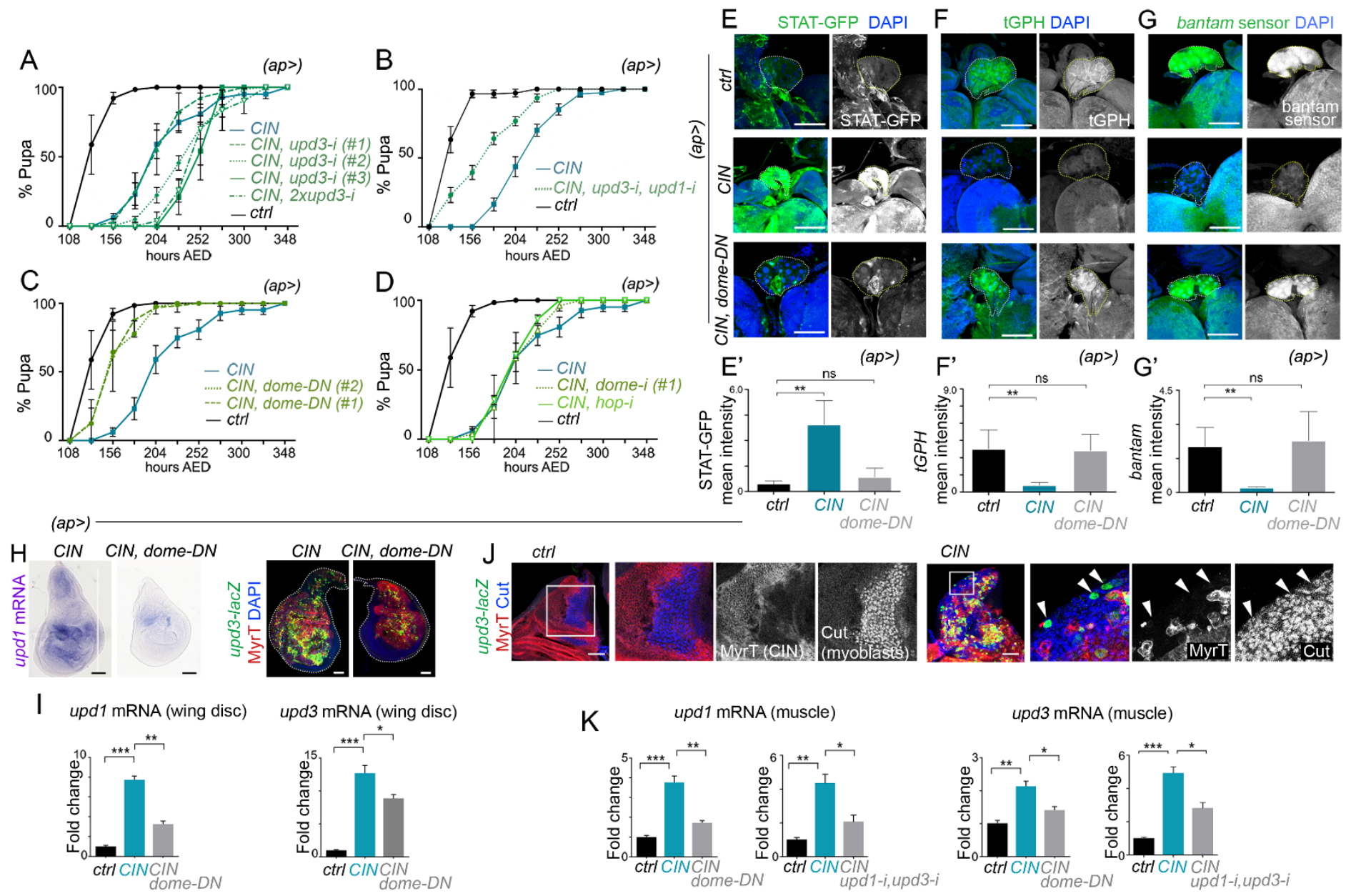

Figure 3. Local co-depletion or trapping of secreted cytokines rescues the CIN-delay.

(A-D) Developmental timing of larvae expressing the indicated transgenes in wing discs with the ap-gal4 driver. (E-G) Ring glands of larvae expressing the indicated transgenes in wing discs (ap-ga/4) and stained for DAPI (blue), STAT-GFP (green or white, E), and tGPH (green or white, F), or bantam sensor (green or white, G). Contour of the ring glands is marked by a dotted line. Scale bars, $100 \mu \mathrm{m}$. (E'-G') Mean signal intensity of STAT-GFP, tGPH and bantam in PGs of larvae expressing the indicated transgenes in wing discs. $(\mathbf{H}, \mathbf{J})$ Wing discs of larvae expressing the indicated transgenes in wing discs with the ap-gal4 driver and stained for upd1 mRNA (purple, $\mathbf{H}$ ), upd3-lacZ (green, $\mathbf{H}, \mathbf{J}$ ), MyrT (red or white, $\mathbf{H}, \mathbf{J}$ ), Cut (blue or white, J, to label myoblasts), and DAPI (blue, H). Scale bars, $50 \mu \mathrm{m}$ (wing discs), $100 \mu \mathrm{m}$ (ring glands). (I, K) upd 1 and upd 3 mRNA levels by qRT-PCR of wing discs (I) and muscle (K) of larvae expressing the indicated transgenes with the ap-gal4 driver. Data represent mean \pm SEM (puparation assays and qRT- 
PCR) or SD (mean intensity). ${ }^{* * *} p<0.001,{ }^{* *} p<0.01,{ }^{*} p<0.05$, ns, non-significant. $n=12-151$ (puparation assays), n=6-12 (mean intensity). AED, after egg deposition. See also Figure S3. 

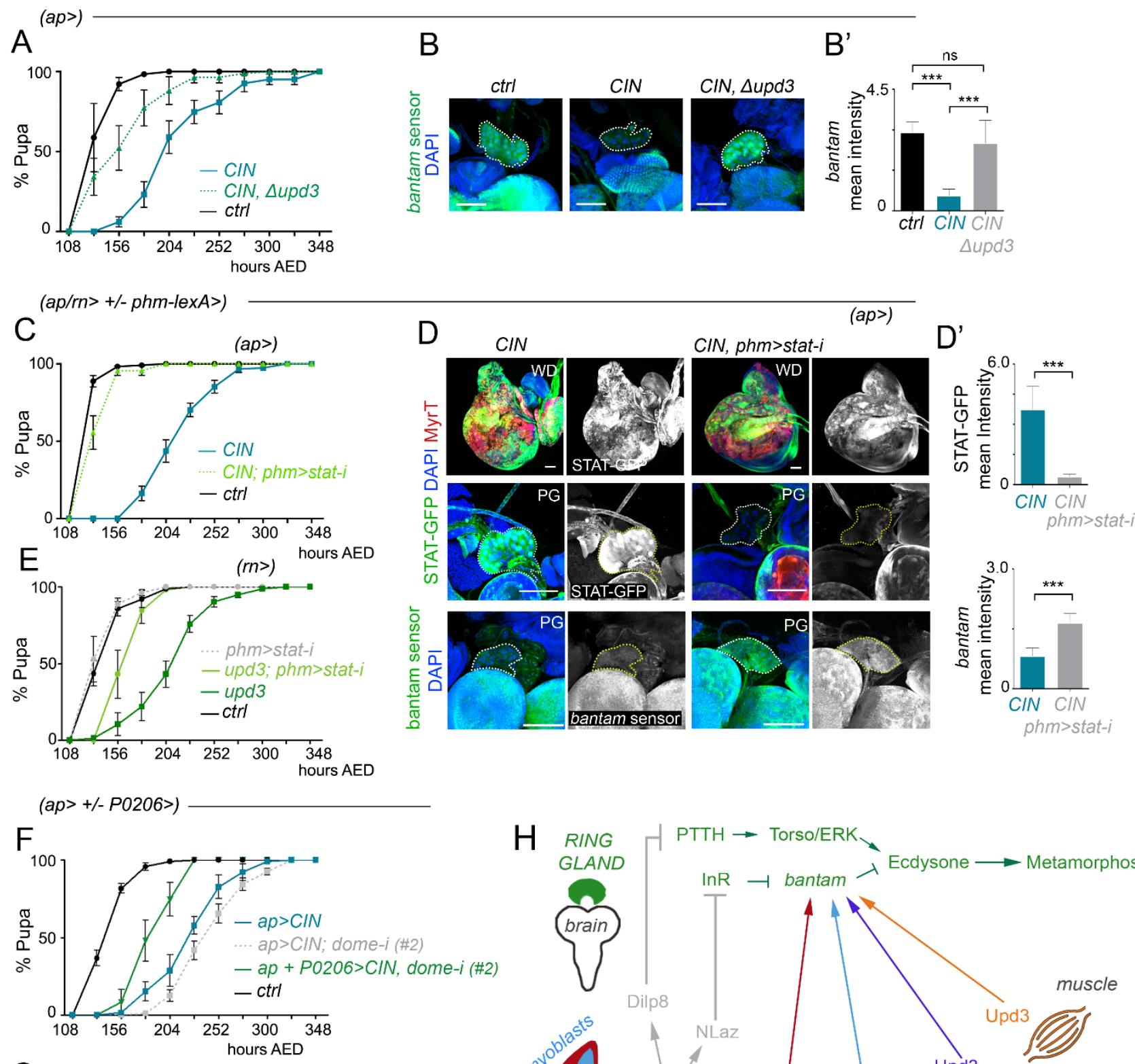

$\mathrm{G}$
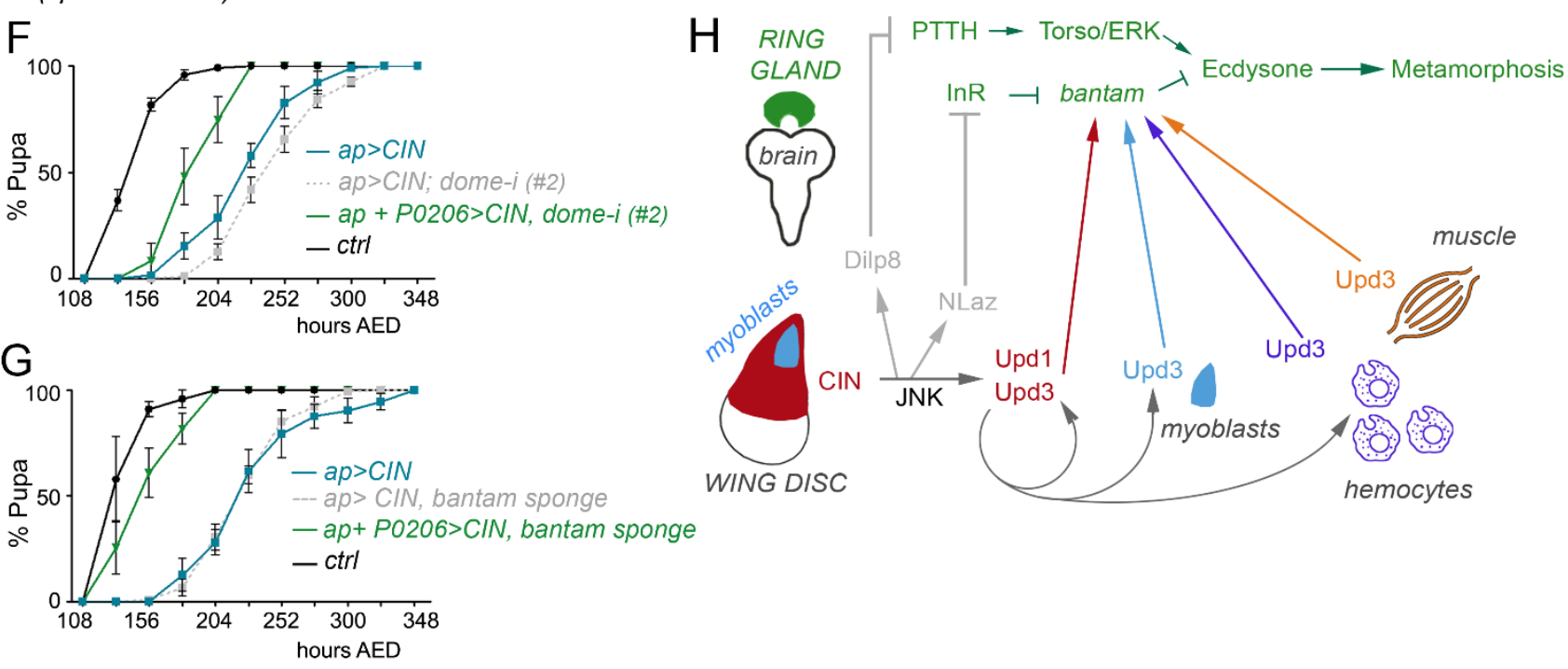

Figure 4. A feed-forward loop spreads upd3 expression to nearby tissues. (A, C, E, F, G). Developmental timing of larvae expressing the indicated transgenes with the ap-gal4 (A, C, F, G), rn-gal4 (E), and P0206-gal4 (F, G) drivers. (B, C) Ring glands of larvae expressing the indicated transgenes in 
wing discs with the ap-gal4 driver and stained for bantam sensor and STAT-GFP (green or white), DAPI (blue) and MyrT (red, D). Scale bars, $100 \mu \mathrm{m}$. (B', D') Mean signal intensity of bantam sensor, and STATGFP in PGs of larvae expressing the indicated transgenes in wing discs and in the PG. In A, B, B', larvae were also mutant for a deletion allele of upd3. In C-E, the ring gland was expressing an RNAi of stat. (H) Schematic representation of the local and systemic contribution of secreted cytokines in the CIN model and their impact on the ecdysone production regulatory network. Data represent mean \pm SEM (puparation assays) or SD (mean intensity). ${ }^{* * *} \mathrm{p}<0.001$, ns, non-significant. $\mathrm{n}=44-194$ (puparation assays), $\mathrm{n}=6-14$ (mean intensity). AED, after egg deposition. See also Figure S4. 
STAR Methods

Key Resources Table

\begin{tabular}{|c|c|c|}
\hline REAGENT or RESOURCE & SOURCE & IDENTIFIER \\
\hline \multicolumn{3}{|l|}{ Antibodies } \\
\hline mouse anti-dMMP1 (14A3D2) & Developmental Studies Hybridoma bank & RRID:AB_579782 \\
\hline rabbit polyclonal anti-GFP (A6455) & Molecular Probe & RRID: AB_221570 \\
\hline mouse polyclonal anti-GFP (A11120) & Molecular Probe & RRID: AB_221568 \\
\hline rabbit anti- $\beta$-galactosidase (0855976) & Cappel (MP Biochemicals) & Code: 0855976 \\
\hline mouse anti-Cut (2B10) & Developmental Studies Hybridoma bank & RRID: AB_528186 \\
\hline rabbit polyclonal anti-p-ERK (4370) & Cell Signaling Technology & RRID:AB_2315112 \\
\hline rat anti-Ci & Developmental Studies Hybridoma bank & RRID:AB_2109711 \\
\hline mouse anti-NimC1 & (Honti et al., 2013) & N/A \\
\hline $\begin{array}{l}\text { Cy2 AffiniPure Donkey Anti-Rabbit IgG } \\
(\mathrm{H}+\mathrm{L})\end{array}$ & Jackson ImmunoResearch & Code: $711-225-152$ \\
\hline $\begin{array}{l}\text { Cy2 AffiniPure Donkey Anti-Mouse IgG } \\
(\mathrm{H}+\mathrm{L})\end{array}$ & Jackson ImmunoResearch & Code: $711-225-151$ \\
\hline $\begin{array}{l}\text { Cy5 AffiniPure Donkey Anti-Mouse IgG } \\
(\mathrm{H}+\mathrm{L})\end{array}$ & Jackson ImmunoResearch & Code: $715-175-150$ \\
\hline $\begin{array}{l}\text { Cy5 AffiniPure Donkey Anti-Rat IgG } \\
(\mathrm{H}+\mathrm{L})\end{array}$ & Jackson ImmunoResearch & Code: $712-175-153$ \\
\hline \multicolumn{3}{|c|}{ Chemicals, Peptides and Recombinant Proteins } \\
\hline DAPI & Sigma Aldrich & Code: $28718-90-3$ \\
\hline 20-Ecdysone & Sigma Aldrich & Code: $5289-74-7$ \\
\hline \multicolumn{3}{|c|}{ Experimental Models. Organisms/Strains } \\
\hline$a p-G A L 4$ & Bloomington Drosophila Stock Center & RRID:BDSC_3041 \\
\hline UAS-myristoylated-Tomato (myrT) & Bloomington Drosophila Stock Center & RRID:BDSC_32222 \\
\hline$U A S-g f p^{R N A i}$ & Bloomington Drosophila Stock Center & RRID:BDSC_35786 \\
\hline
\end{tabular}




\begin{tabular}{|c|c|c|}
\hline elav-Gal80 & Bloomington Drosophila Stock Center & RRID:BDSC_98193 \\
\hline UAS- $w g^{R N A i}$ (\#1 in the text) & Bloomington Drosophila Stock Center & RRID:BDSC_32994 \\
\hline UAS- $w g^{R N A i}(\# 2$ in the text) & Bloomington Drosophila Stock Center & RRID:VDRC_104579 \\
\hline dilp8mimic-GFP & Bloomington Drosophila Stock Center & RRID:BDSC_33079 \\
\hline UAS-p35 & Bloomington Drosophila Stock Center & RRID:BDSC_5073 \\
\hline UAS-BskDN & Bloomington Drosophila Stock Center & RRID:BDSC_6409 \\
\hline en-GAL4 & Bloomington Drosophila Stock Center & RRID:BDSC_1973 \\
\hline$\Delta u p d 3$ & Bloomington Drosophila Stock Center & RRID: BDSC_55728 \\
\hline UAS-upd1 $1^{R N A i}$ & Bloomington Drosophila Stock Center & RRID: BDSC 28722 \\
\hline UAS-upd $3^{R N A i}$ (\#1 in the text) & Bloomington Drosophila Stock Center & RRID: BDSC_28575 \\
\hline UAS-upd $3^{R N A i}$ (\#2 in the text) & VDRC Stock Center & RRID: VDRC_27136 \\
\hline UAS-upd $3^{R N A I}$ (\#3 in the text) & VDRC Stock Center & RRID: VDRC_27134 \\
\hline phm-gal4 & Bloomington Drosophila Stock Center & RRID: BDSC_80577 \\
\hline$U A S-b u b 3^{R N A i}$ & VDRC Stock Center & RRID:VDRC_21037 \\
\hline UAS-dilp8 $8^{R N A i}$ (\#1 in the text) & VDRC Stock Center & RRID: VDRC_9420 \\
\hline UAS-dilp8 $8^{R N A i}$ (\#2 in the text) & VDRC Stock Center & RRID: VDRC_102604 \\
\hline $\operatorname{dilp} 8^{K O}$ & (Boone et al., 2016) & $\mathrm{N} / \mathrm{A}$ \\
\hline UAS-PTTH & (Yamanaka et al., 2013) & $\mathrm{N} / \mathrm{A}$ \\
\hline UAS-rod ${ }^{R N A i}$ & VDRC Stock Center & RRID:VDRC_16152 \\
\hline UAS-NLaz ${ }^{R N A i}(\# 1$ in the text) & VDRC Stock Center & RRID:VDRC_107553 \\
\hline UAS-NLaz ${ }^{R N A i}$ (\#2 in the text) & VDRC Stock Center & RRID:VDRC_35558 \\
\hline UAS-NLaz & Bloomington Drosophila Stock Center & RRID:BDSC_76608 \\
\hline$N L a z^{N W 5} / C y O$ & Bloomington Drosophila Stock Center & RRID:BDSC_76609 \\
\hline UAS-avalanche ${ }^{R N A i}$ & VDRC Stock Center & RRID:VDRC_107264 \\
\hline UAS-puc & (Martin-Blanco et al., 1998) & $\mathrm{N} / \mathrm{A}$ \\
\hline bantam sensor (II) & (Brennecke et al., 2003) & $\mathrm{N} / \mathrm{A}$ \\
\hline bantam sensor (III) & (Brennecke et al., 2003) & $\mathrm{N} / \mathrm{A}$ \\
\hline
\end{tabular}




\begin{tabular}{|c|c|c|}
\hline UAS-bantam-sponge & (Becam et al., 2011) & $\mathrm{N} / \mathrm{A}$ \\
\hline tGPH sensor & (Britton et al., 2002) & N/A \\
\hline UAS-dilp8 & (Colombani et al., 2012) & N/A \\
\hline upd3-lacZ (II) & (Bunker et al., 2015) & $\mathrm{N} / \mathrm{A}$ \\
\hline STATGFP10x (II) & (Bach et al., 2007) & N/A \\
\hline STATGFP10x (III) & Bloomington Drosophila Stock Center & RRID:BDSC_26198 \\
\hline UAS-hop & (Sotillos et al., 2008) & N/A \\
\hline UAS-upd3 & (Wang et al., 2014) & $\mathrm{N} / \mathrm{A}$ \\
\hline UAS-domeDN (III) (\#1 in the text) & (Brown et al., 2001) & N/A \\
\hline UAS-domeDN (II) (\#2 in the text) & (Brown et al., 2001) & $\mathrm{N} / \mathrm{A}$ \\
\hline P0206-gal4 & kindly given by H. Jasper & $\mathrm{N} / \mathrm{A}$ \\
\hline UAS-hOp ${ }^{R N A i}$ & Bloomington Drosophila Stock Center & RRID:BDSC_31319 \\
\hline UAS-dome ${ }^{R N A i}(\# 1$ in the text) & VDRC Stock Center & RRID:VDRC_106071 \\
\hline UAS-dome ${ }^{R N A i}(\# 2$ in the text) & VDRC Stock Center & RRID:VDRC_36356 \\
\hline mef2-gal4 & VDRC Stock Center & RRID:VDRC_50742 \\
\hline GMR15B03-gal4 & Bloomington Drosophila Stock Center & RRID:BDSC_49261 \\
\hline UAS-upd1 & (Ayala-Camargo et al., 2013) & N/A \\
\hline hemese-gal4 & Bloomington Drosophila Stock Center & RRID:BDSC_8699 \\
\hline croquemort-gal4 & Bloomington Drosophila Stock Center & RRID:BDSC_25041 \\
\hline$c g-g a l 4$ & Bloomington Drosophila Stock Center & RRID:BDSC_7011 \\
\hline phm-lexA & kindly given by Gary Struhl & N/A \\
\hline upd3-gal4 & (Agaisse et al., 2003) & N/A \\
\hline LexAop stat-i & generated in this work & $\mathrm{N} / \mathrm{A}$ \\
\hline \multicolumn{3}{|l|}{ Software and Algorithms } \\
\hline Fiji & Fiji & https://fiji.sc/ \\
\hline Excel & Microsoft Excel 2016 & N/A \\
\hline GraphPad Prism 7 Project & GraphPad & RRID:SCR_002798 \\
\hline
\end{tabular}




\section{Contact for Reagent and Resource Sharing}

Further information and requests for resources and reagents should be directed to and will be fulfilled by the Lead Contact, Marco Milán (marco.milan@irbbarcelona.org).

\section{Experimental Model and Subject Details}

\section{Fly strains}

The strains used were kindly provided by the following sources: (1) Bloomington Drosophila Stock Center (BDSC): ap-GAL4; UAS-myristoylated-Tomato; UAS-gfp ${ }^{R N A i}$; elav-Gal80; UAS-wg ${ }^{R N A i} \# 1$; UAS-wg ${ }^{R N A i}$ \#2; dilp8mimic-GFP; UAS-NLaz ${ }^{N W 5} / C y O ; ~ U A S-p 35 ;$ UAS-bskDN; en-GAL4; 10XSTAT-GFP(III); $\Delta$ upd3; UASupd1RNAi; UAS-Upd3 $^{R N A i} \# 1$;; UAS-hop ${ }^{R N A i} ;$ phm-GAL4; hem-GAL4; crq-GAL4; GMR15B03- GAL4; cgGAL4; (2) Vienna Drosophila RNAi Center (VDRC): UAS-bub3 ${ }^{\text {RAAi }}$ UAS-dilp8 $8^{R N A i}$ \#1; UAS-dilp8 ${ }^{R N A i}$ \#2;

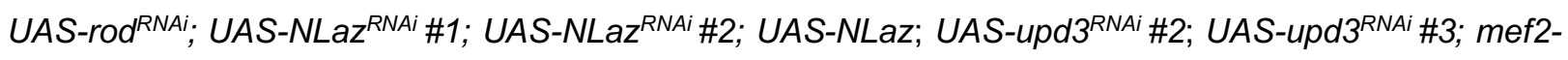
gal4; UAS-avalanche ${ }^{R N A i}$; UAS-dome $e^{R N A i} \# 1$; UAS-dome ${ }^{R N A i} \# 2$, and (3) UAS-puc (J. P. Vincent); bantam

sensor (S. Cohen); $t G P H$ sensor (H. Stocker); UAS-dilp8, dilp8 ${ }^{K O}$ (P. Leopold); upd3-lacZ (H. Jiang); 10XSTAT-GFP (II) (M. Dominguez), UAS hop (J. Castelli), UAS-upd3 (D. Harrison), upd3-gal4 (B. Lemaitre), P0206-gal4 (H. Jasper), UAS-domeDN \#1 and \#2 (J. Castelli), UAS-upd1 (E. Bach), UASPTTH (F. Martín), and phm-lexA (G. Struhl). Flies were allowed to lay eggs on fly food for $12 \mathrm{~h}$ at $25^{\circ} \mathrm{C}$ for dissection assays and $5 \mathrm{~h}$ at $25^{\circ} \mathrm{C}$ for pupariation assays. Larvae were then switched to $29^{\circ} \mathrm{C}$ for 4 days before dissection or pupa counts took place.

\section{Method Details}

$\underline{\text { Immunohistochemistry and confocal imaging }}$

Wing imaginal discs, brains, ring glands, trachea and fat body tissues of third instar larvae were dissected in cold PBS, fixed in 4\% formaldehyde for 20 min and immunolabeled in BBT (PBS with $0.3 \%$ BSA, 0.2\% Triton X-100) with the following antibodies: mouse anti-MMP1 (1:20) (14A3D2, Developmental Studies Hybridoma Bank, DSHB); rat anti-Ci (1:10; 2A1, DSHB); rabbit and mouse anti-GFP (1:600) (Molecular 
Probe, A6455 and A11120, respectively); rabbit anti- $\beta$-galactosidase (1:600) (Cappel; 0855976); mouse anti-Cut (1:100) (2B10, Developmental Studies Hybridoma Bank, DSHB), rabbit polyclonal anti-p-ERK (1:100) (4370, Cell Signaling Technology), mouse anti-NimC1 (1:25) kindly provided by I. Andó. For the muscle tissues, the general procedure was the same as described previously except for an extended time of fixation of $30 \mathrm{~min}$ after filet dissection after which the 10xSTAT-GFP reporter was analyzed. Secondary antibodies Cy2 and Cy5 (1:400) were obtained from Jackson ImmunoResearch. Leica TCS SP5 MP confocal microscope was used to perform high resolution images. NLaz digoxigenin (DIG)-labelled antisense probe was transcribed by T3 RNA polymerase from a Xhol-linearized plasmid containing the fulllength cDNA (clone RE67583 obtained from DGRC). upd1 DIG-labelled antisense probe was transcribed by T7 polymerase from an EcoRI-linearized plasmid containing a 600bp fragment of upd1 cDNA (nt 946 to 1547, this work). Probes were synthesized using the DIG RNA Labelling Kit (Roche) according to the manufacturer's instructions. In situ hybridization protocol was performed as in (Milan et al., 1996).

\section{LexAop-stat-RNAi cloning}

To generate the LexAop-statRNAi construct, the following oligos were used. stat-i Fwd (EcoRI)

CTA GCA GTT CGC AGC TTT ATG TAC GAT AGT TAT ATT CAA GCA TAT CGT AGT ACA AGC TGC GAG CG

stat-i Rev (Xbal)

AAT TCG CTC GCA GCT TTA TGT ACT ACG ATA TGC TTG AAT ATA ACT ATC GTA CAT AAA GCT GCG AAC TG

$9.5 \mu$ l of each oligo $(100 \mu \mathrm{M})$ was used to mix with $1 \mu \mathrm{l}$ of annealing buffer (20X SSC). The mix was heated at $100^{\circ} \mathrm{C}$ for 10 minutes and let to cool down overnight for an efficient annealing. 1/200 dilution of the annealed mix was used to ligate in the pLOT plasmid (carrying the lexA operator), previously digested with EcoRI and Xbal endonucleases.. The plasmid was transformed in DH5alpha competent cells and 5 colonies were selected for checking. To assess for the correct generation of vector and oligos combined, the absence of the $\mathrm{Xhol}_{3461 \mathrm{bp}}$ digestion site was checked. Plasmids with the correct size and absence of the Xhol site were sequenced and one positive clone was used to generate transgenic flies with standard protocols. 


\section{Pupariation Assays}

Flies were allowed to lay eggs for $4 \mathrm{~h}$ (or a maximum of $5 \mathrm{~h}$ for difficult phenotypes, namely the mutants) at $25^{\circ} \mathrm{C}$ after which they were switched to $29^{\circ} \mathrm{C}$ for a total of 4 days. After these 4 days of induction, tubes were taken from the bath and placed at room temperature to allow for counting from 108 to $358 \mathrm{~h}$ after egg deposition, accounting for a period of approximately referent from 5 to 15 days' time. Counts were performed always at the same time point and $24 \mathrm{~h}$ apart. The resulting percentage of pupae was calculated accordingly with the total number of larvae capable of transitioning to the next developmental phase.

\section{Larval Feeding Assays}

For the blue food feeding assays, flies were allowed to lay eggs for a maximum of $5 \mathrm{~h}$ at $25^{\circ} \mathrm{C}$ and were then switched to $29^{\circ} \mathrm{C}$ for 4 days. After this induction period larvae were transferred to plates with food supplemented with Bromophenol Blue (Sigma-Aldrich 114391_5G). Next, L3 larvae were isolated from each plate at different time points. The presented data refer to the highly relevant time points of $132 \mathrm{~h}, 156 \mathrm{~h}$, 180h and 204h imaged using an Inverted Microscope Olympus IX71 SCMOS.

\section{Ecdysone Feeding Assays}

Flies were allowed to lay eggs on standard ecologic fly food for $4 \mathrm{~h}$ at $25^{\circ} \mathrm{C}$ and were then switched to $29^{\circ} \mathrm{C}$. At $84 \mathrm{~h}$ they were selected for early L3 stage characteristics and switched to food supplemented with an activated form of ecdysone. Stock solution for the steroidal hormone at a concentration of $5 \mathrm{mg} / \mathrm{ml}$ was prepared from $5 \mathrm{mg}$ of 20 -ecdysone powder (Sigma) in $1 \mathrm{~mL}$ of EtOH. Working solution was adjusted by diluting the initial stock solution in PBS until a final concentration of $0.2 \mathrm{mg} / \mathrm{ml}$. Control solutions were prepared using the same amount of EtOH in PBS. The amount of hormone and control solution were calculated to allow for the dilution to add up to a total volume of $100 \mathrm{ml}$. Each dilution was prepared daily and $50 \mathrm{ml}$ was added first thing in the morning and last thing in the afternoon, respecting a 10-h window. The food in each vial was stirred slightly prior to addition of the hormone or control solution to facilitate diffusion.

\section{Quantitative RT-PCR}


For the quantification of mRNA levels, total RNA was extracted from wing discs and muscles of at least 25 and 15 larvae, respectively, using TRIzol reagent (Invitrogent). A total of $1.5 \mu \mathrm{g}$ of total RNA was treated with DNase and used as a template for cDNA synthesis using Maxima Reverse Transciptase (ThermoScientific). Maxima SYBR Green/ROX qPCR Master Mix (ThermoScientific) was used, and reactions were run in a QuantStudio 6 Pro Real-Time PCR Systems (Applied Biosystems). Samples were normalized to the levels of forkhead domain 68A (Mnf, for wing disc samples), or Actin42A (Act, for muscle samples) transcript levels and fold changes were calculated using the $\Delta \Delta \mathrm{Ct}$ method. Three separate biological samples were collected for each experiment. The following primer pairs were used: Mnf-Fw: 5'GAG CAG AAG AGC CCC TAC CT-3', Mnf-Rv: 5'-AAT GAA ACC CTG ACG TGG AC-3' (Ponton et al., 2011); Act-Fw: 5'-GCG TCG GTC AAT TCA ATC TT-3', Act-Rv: 5'-AAG CTG CAA CCT CTT CGT CA3'(Ponton et al., 2011); Dilp8-Fw: 5'- GCA CCA CCA TCT GAA TCG AC-3', Dilp8-Rv: 5'- CTG AGG CGA TTG AAG TGC TC -3'; NLaz-Fw:5'- GCC AGA AGT AGA ACG GAT ACC A-3', NLaz-Rv:5'- ACT GGT GCA GCT GTA GAC GAC-3' (Hull-Thompson et al., 2009); Upd1-Fw: 5'- AGA CAG CCG TCA ACC AGA C-3', Upd1-Rv: 5'- AGA CAG CCG TCA ACC AGA C-3' [(Moskalev et al., 2019), wing disc samples)]; Upd1-Fw: 5'- TGT AAC CCC GTT CGC TGT AT-3', Upd1-Rv: 5'- GCT GAT GTT TCC GTT TCC GT-3' (muscle samples); Upd3-Fw: 5'- ATC CCA CCA ATC CCC TGA AG-3', Upd3-Rv: 5'-AGA TTG CAG GTG TTC TCC CA-3'.

Quantification and Statistical Analysis

$\underline{\text { Image Processing and Analysis }}$

Fiji [National Institute of Health (NIH) Bethesda, MD] was used to measure the size of the anterior (a), dorsal (d) compartments (based on Ci and MyrT expression respectively), or the whole wing discs (based on DAPI staining). Image stacks were obtained using a Leica TCS SP5 MP confocal microscope, 20X dry objective with $1 \mu \mathrm{m}$ per optical section to cover the entire thickness of each disc. Maximum intensity Zprojection was performed on the stacks prior to reporter analysis. Control wing discs grown in parallel and subjected to the same experimental conditions (temperature and time of transgene induction) were analyzed in parallel. At least 10 wing discs per genotype were scored. In the special case of the myoblast 
population, a 40x glycerol immersion objective was used to facilitate the acquisition of high-quality images of both tumor and myoblast cells.

Fiji [National Institute of Health (NIH) Bethesda, MD] was also used to perform mean intensity measurements of signaling reporters in the ring gland ( $\mathrm{pERK}, \mathrm{tGPH}$, bantam-sensor, STAT-GFP) by creating a selection of the ring gland tissue and measuring associated mean grey values. This measurement accounts for the mean pixel intensity associated with that fluorescence against a minimum threshold defined to avoid nonspecific signal staining.

\section{Statistical Analysis}

Statistical analysis was generally performed by unpaired equal-variance two-tail t-test. Differences were considered significant when $p$ values were less than $0.001\left(^{* * *}\right), 0.01\left(^{* *}\right)$, or $0.05\left(^{*}\right)$. All genotypes included in each histogram were analyzed in parallel. All data points were plotted in Prism 7.0 (Graphpad) statistical software. 


\section{Supplementary Figures}

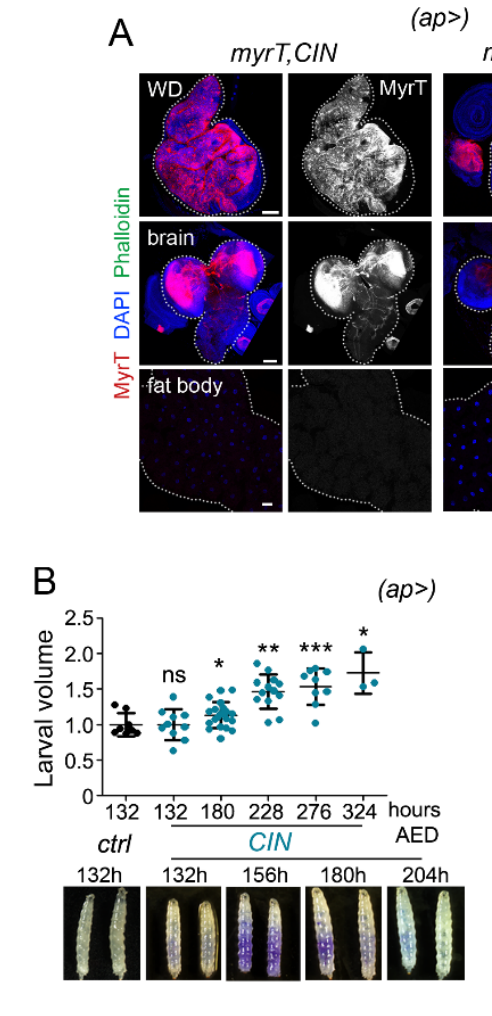

$(a p>)$
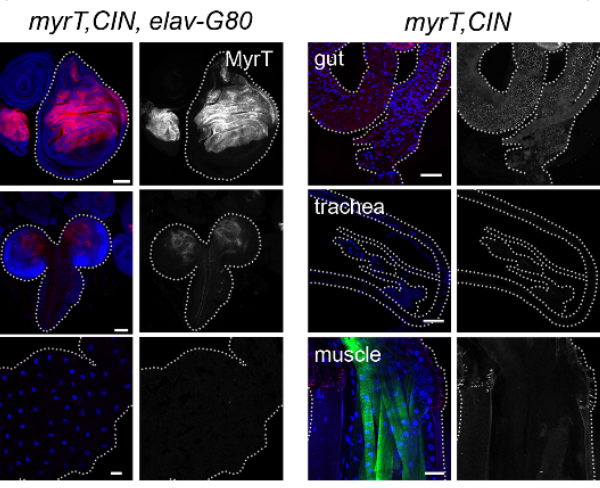

$(a p>)$
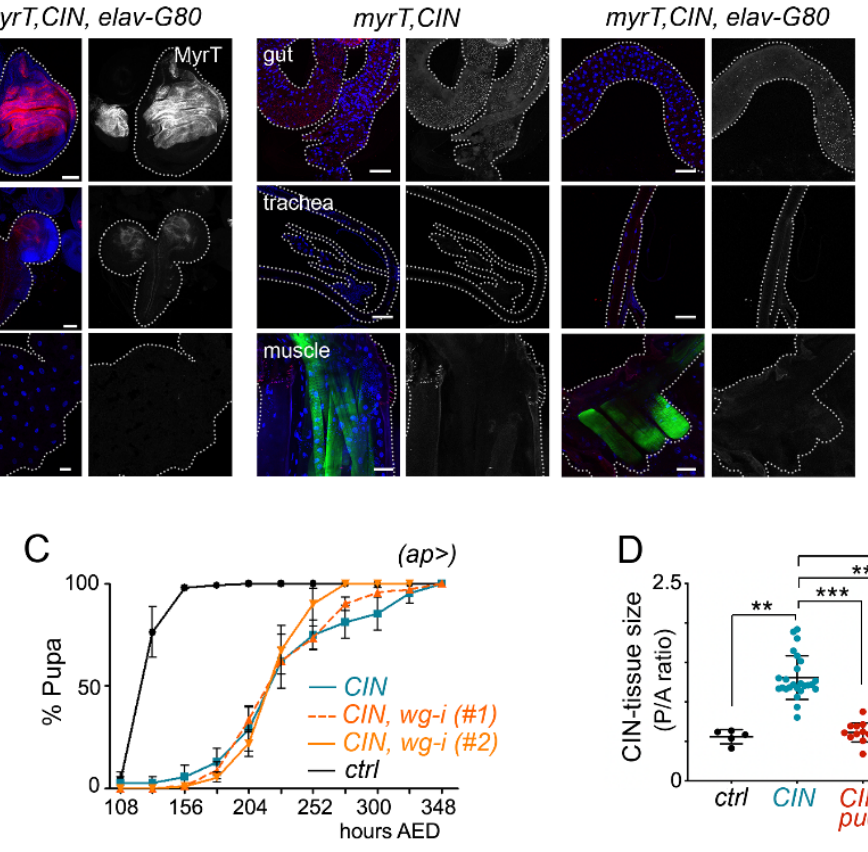

$(e n>)$
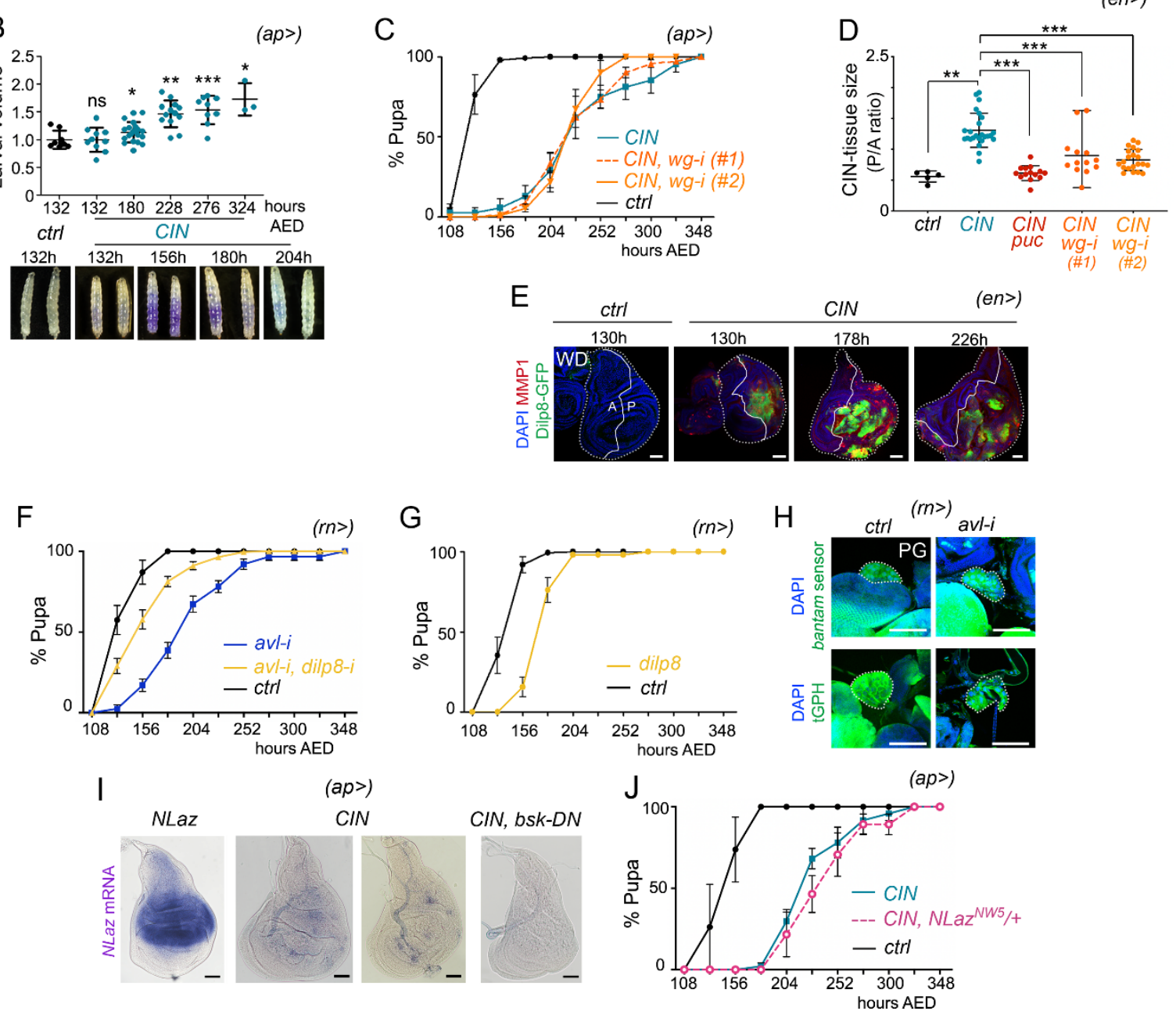

Figure S1. CIN induces a Dilp8-independent developmental delay (related to Figure 1) 
(A) Wing discs, brain, fat body, gut, trachea and muscle tissues (contours depicted by a dotted line) expressing the indicated transgenes under the control of ap-gal4 and stained for MyrT (red), DAPI (blue) and phalloidin (green). MyrT labels the ap-gal4 domain (red). (B) Larval volume and feeding behavior of larvae expressing the indicated transgenes under the control of ap-gal4. Data represent mean \pm SD. NS, non-significant, ${ }^{* * *} p<0.001,{ }^{* *} p<0.01,{ }^{*} p<0.05$. $n=3-20$. (C, F, G, J) Developmental timing of larvae expressing the indicated transgenes in wing discs with ap-ga/4 (C, J) or rn-ga/4 $(\mathbf{F}, \mathbf{G})$. In $\mathbf{J}$, larvae were also heterozygous for NLaz. $n=50-221$, represent mean \pm SEM. (D) Tissue size of wing discs (P/A ratio) subjected to expression of the indicated transgenes in the posterior $(P)$ compartment. Data represent mean $\pm S D .{ }^{* *} p<0.001,{ }^{* *} p<0.01,{ }^{*} p<0.05 ; n=5-25$. (E) Wing discs expressing the indicated transgenes under the control of en-gal4 driver and stained with DAPI (blue), MMP1 (red) and Dilp8-GFP (green). The anteriorposterior (AP) boundary is depicted by a white line and wing disc contours by a dotted line. (H) Ring glands of larvae expressing the indicated transgenes in wing discs with rn-gal4 and stained for DAPI (blue) and tGPH or bantam sensors (green). (I) Wing imaginal discs expressing the indicated transgenes with ap-gal4 and stained for NLaz mRNA expression (purple). Scale bars, $50 \mu \mathrm{m}$ (wing discs), $100 \mu \mathrm{m}$ (ring glands). AED, after egg deposition. 

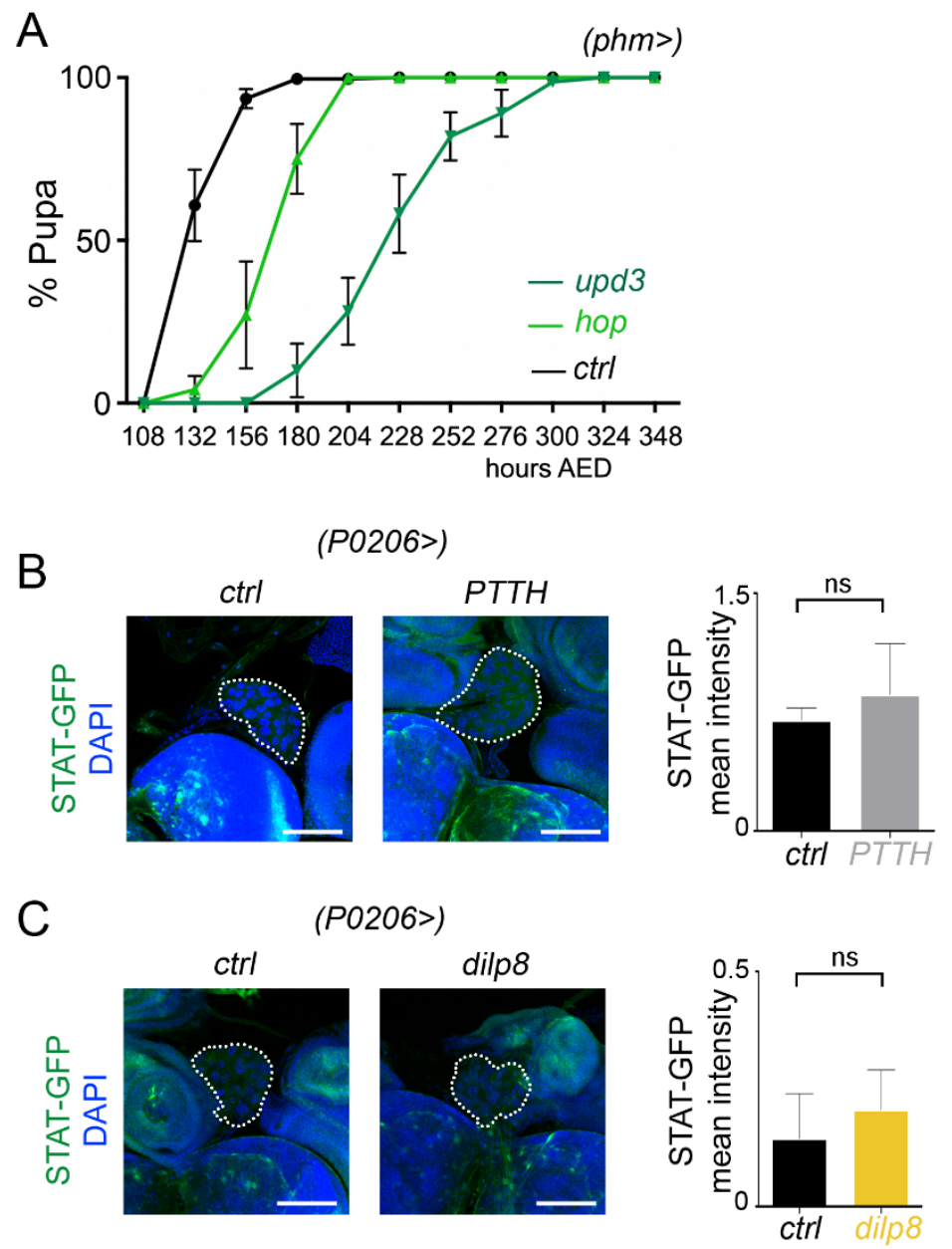

Figure S2. Local activation of JAK/STAT in the prothoracic gland induces a developmental delay (related to Figure 2)

(A) Developmental timing of larvae expressing the indicated transgenes in the prothoracic gland with phmgal4. (B, C) On the left, ring glands of larvae expressing the indicated transgenes in the PG (P0206-gal4), and stained for STAT-GFP (green) and DAPI (blue). On the right, mean signal intensity of STAT-GFP in PGs of larvae expressing the indicated transgenes in the PG (P0206-gal4). Scale bars, $100 \mu \mathrm{m}$. Data represent mean \pm SEM (puparation assays) or SD (mean intensity). ns, non-significant. $\mathrm{n}=28-128$ (puparation assays), $\mathrm{n}=7-10$ (mean intensity). AED, after egg deposition. 

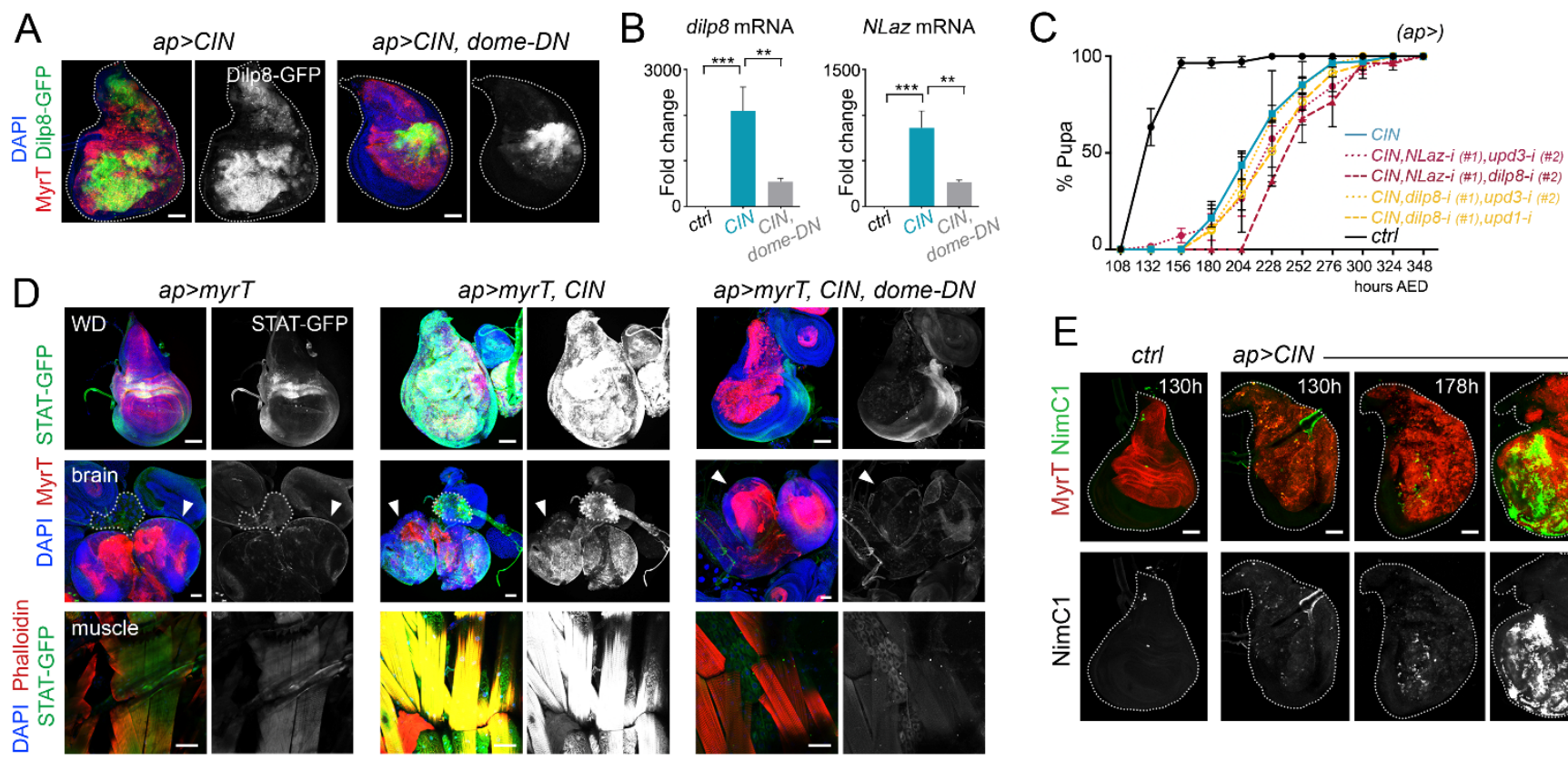

$E$

hours AED

$\mathrm{F}$

G
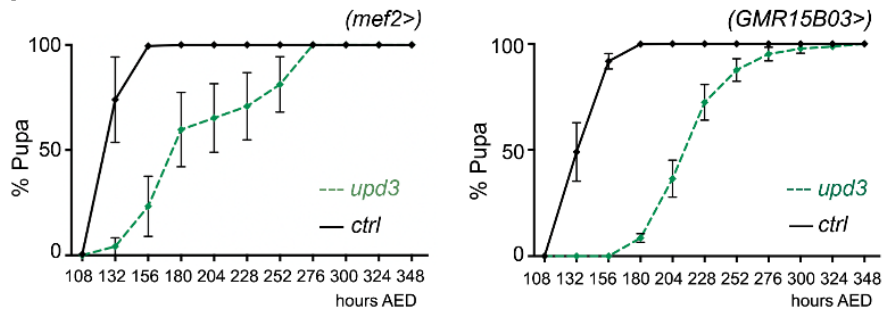

$\mathrm{H}$
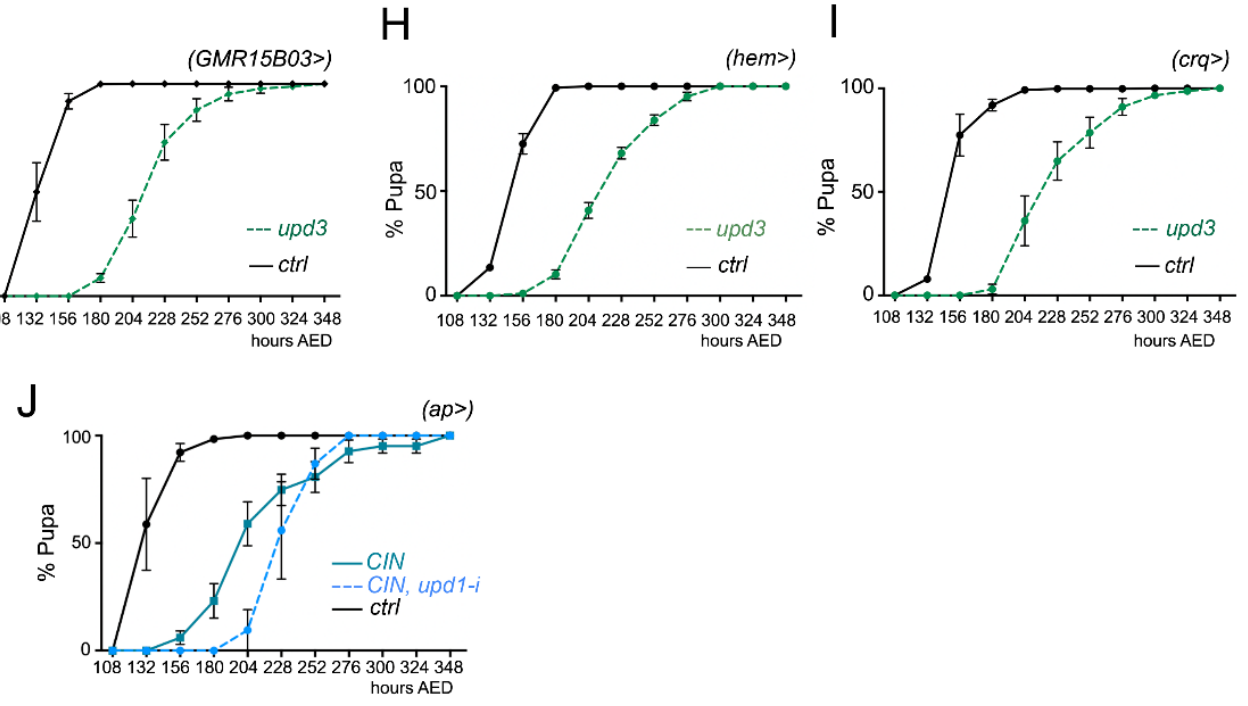

Figure S3. Depletion of upd1 is not sufficient to rescue the CIN-developmental delay (related to Figure 3)

(A, D, E) Wing discs, brain (white arrowhead in D) and muscle (D) tissues of larvae expressing the indicated transgenes under the control of ap-gal4 driver and stained for MyrT (red), DAPI (blue, A, D), Dilp8-GFP (green or white, A), STAT-GFP (green or white, D) and NimC1 (green or white, E). Scale bars, $50 \mu m$. (B) dilp8 and NLaz mRNA levels by qRT-PCR of wing discs expressing the indicated transgenes with the ap- 
gal4 driver. Contour of wing discs and ring glands is marked by a dotted line. (C, F-J) Developmental timing of larvae expressing the indicated transgenes in wing disc (ap-gal4, C, J), muscles (mef2-gal4, F), myoblasts (GMR15B03-gal4, G), or hemocytes (hem-gal4, H, crq-gal4, I). Data represent mean \pm SEM (puparation assays and qRT-PCR), $n=12-447$ (puparation assays). ${ }^{* * *} p<0.001,{ }^{* *} p<0.01$. AED, after egg deposition. 
A

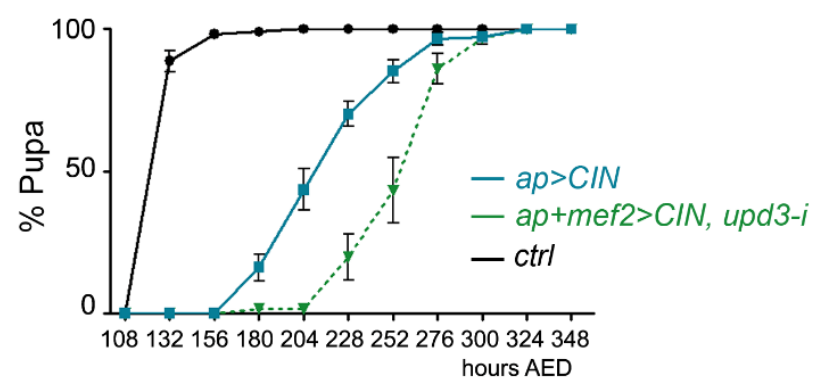

B
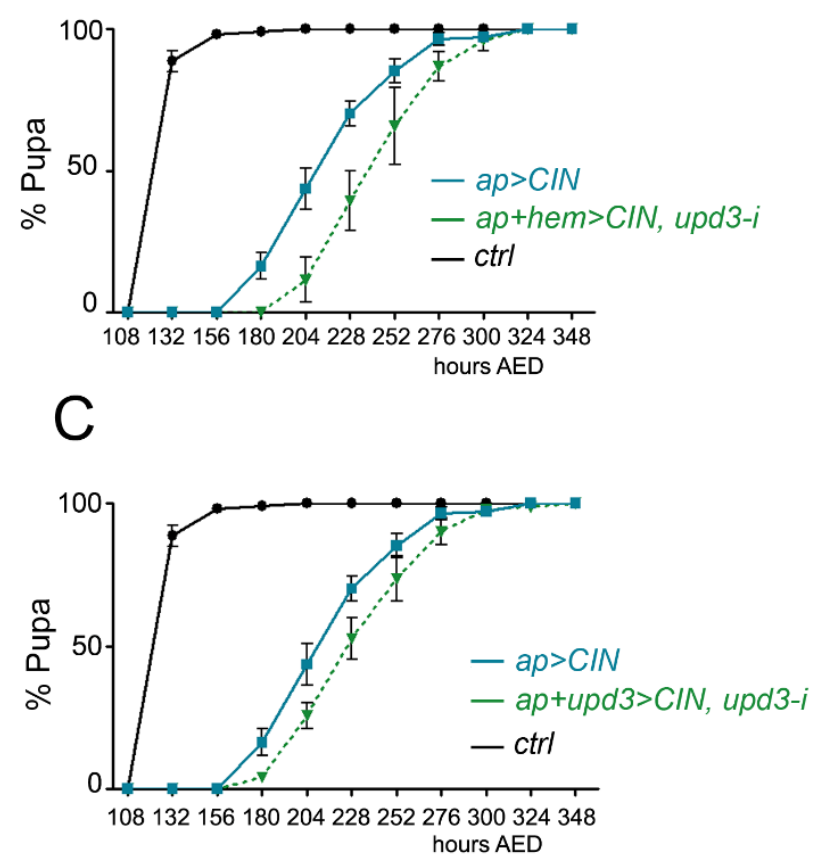

D

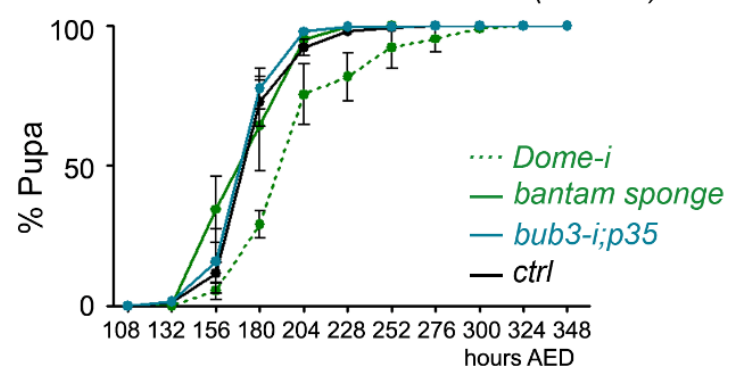

E

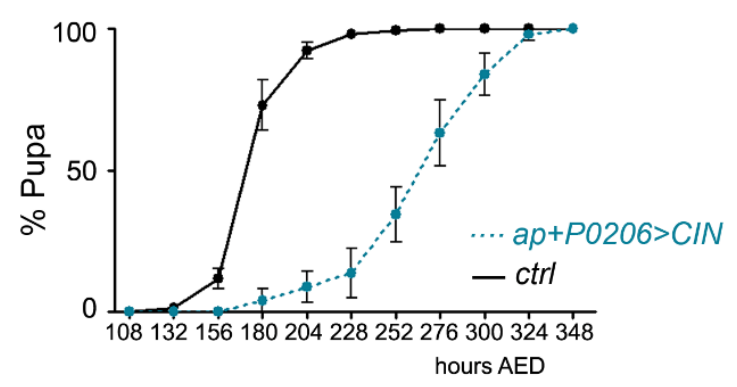

$\mathrm{F}$

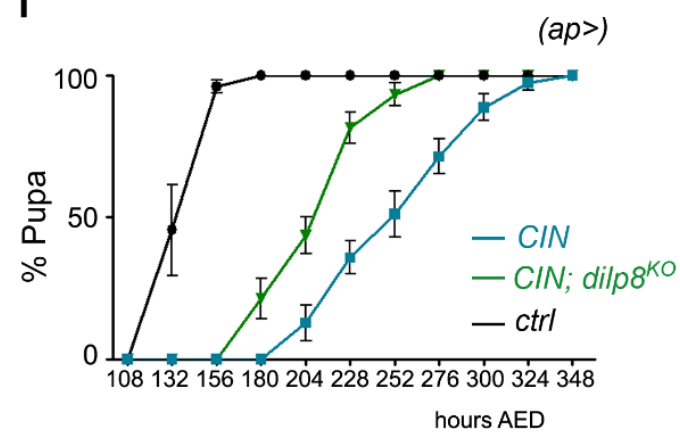

Figure S4. Different sources of Upd3 contribute to the CIN-developmental delay (related to Figure 4)

(A-F) Developmental timing of larvae expressing the indicated transgenes in wing disc (ap-gal4), muscles (mef2-gal4), hemocytes (hem-gal4, upd3-gal4) and ring gland (P0206-gal4). Data represent mean \pm SEM $(n=20-447)$. AED, after egg deposition. 


\section{References}

Agaisse, H., Petersen, U.M., Boutros, M., Mathey-Prevot, B., and Perrimon, N. (2003). Signaling role of hemocytes in Drosophila JAK/STAT-dependent response to septic injury. Dev. Cell.

Andersen, D.S., Colombani, J., and Léopold, P. (2013). Coordination of organ growth: principles and outstanding questions from the world of insects. Trends Cell Biol. 23, 336-344.

Ayala-Camargo, A., Anderson, A.M., Amoyel, M., Rodrigues, A.B., Flaherty, M.S., and Bach, E.A. (2013). JAK/STAT signaling is required for hinge growth and patterning in the Drosophila wing disc. Dev Biol 382, 413-426.

Bach, E.A., Ekas, L.A., Ayala-Camargo, A., Flaherty, M.S., Lee, H., Perrimon, N., and Baeg, G.H. (2007). GFP reporters detect the activation of the Drosophila JAK/STAT pathway in vivo. Gene Expr Patterns 7, 323-331.

Ballinger, A.B., Savage, M.O., and Sanderson, I.R. (2003). Delayed Puberty Associated with Inflammatory Bowel Disease. Pediatr. Res. 53, 205-210.

Becam, I., Rafel, N., Hong, X., Cohen, S.M.M., Milán, M., and Milan, M. (2011). Notch-mediated repression of bantam miRNA contributes to boundary formation in the Drosophila wing. Development 138, 3781-3789.

Boone, E., Colombani, J., Andersen, D.S., and Leópold, P. (2016). The Hippo signalling pathway coordinates organ growth and limits developmental variability by controlling dilp8 expression. Nat. Commun.

Boulan, L., Martín, D., and Milán, M. (2013). bantam miRNA Promotes Systemic Growth by Connecting Insulin Signaling and Ecdysone Production. Curr. Biol. 23, 473-478.

Brain, C.E., and Savage, M.O. (1994). Growth and puberty in chronic inflammatory bowel disease. Baillieres. Clin. Gastroenterol. 8, 83-100.

Brennecke, J., Hipfner, D.R., Stark, A., Russell, R.B., and Cohen, S.M. (2003). bantam encodes a developmentally regulated microRNA that controls cell proliferation and regulates the proapoptotic gene hid in Drosophila. Cell 113, 25-36.

Britton, J.S.S., Lockwood, W.K.K., Li, L., Cohen, S.M.M., and Edgar, B.A.A. (2002). Drosophila's insulin/PI3-kinase pathway coordinates cellular metabolism with nutritional conditions. Dev Cell 2, 239249.

Brown, S., Hu, N., and Hombria, J.C. (2001). Identification of the first invertebrate interleukin JAK/STAT 
receptor, the Drosophila gene domeless. Curr Biol 11, 1700-1705.

Bunker, B.D., Nellimoottil, T.T., Boileau, R.M., Classen, A.K., and Bilder, D. (2015). The transcriptional response to tumorigenic polarity loss in Drosophila. Elife 4.

Clemente-Ruiz, M., Murillo-Maldonado, J.M., Benhra, N., Barrio, L., Pérez, L., Quiroga, G., Nebreda, A.R., and Milán, M. (2016). Gene Dosage Imbalance Contributes to Chromosomal Instability-Induced Tumorigenesis. Dev. Cell 36, 290-302.

Colombani, J., Bianchini, L., Layalle, S., Pondeville, E., Dauphin-Villemant, C., Antoniewski, C., Carré, C., Noselli, S., and Léopold, P. (2005). Antagonistic actions of ecdysone and insulins determine final size in Drosophila. Science 310, 667-670.

Colombani, J., Andersen, D.S.D.S., Leopold, P., and Léopold, P. (2012). Secreted peptide Dilp8 coordinates Drosophila tissue growth with developmental timing. Science 336, 582-585.

Colombani, J., Andersen, D.S., Boulan, L., Boone, E., Romero, N., Virolle, V., Texada, M., and Léopold, P. (2015). Drosophila Lgr3 Couples Organ Growth with Maturation and Ensures Developmental Stability. Curr. Biol. 25, 2723-2729.

Dekanty, A., Barrio, L., Muzzopappa, M., Auer, H., and Milan, M. (2012). Aneuploidy-induced delaminating cells drive tumorigenesis in Drosophila epithelia. Proc. Natl. Acad. Sci. 109, 20549-20554.

Garelli, A., Gontijo, A.M., Miguela, V., Caparros, E., and Dominguez, M. (2012). Imaginal Discs Secrete Insulin-Like Peptide 8 to Mediate Plasticity of Growth and Maturation. Science (80-. ). 336, 579-582.

Garelli, A., Heredia, F., Casimiro, A.P., Macedo, A., Nunes, C., Garcez, M., Dias, A.R.M., Volonte, Y.A., Uhlmann, T., Caparros, E., et al. (2015). Dilp8 requires the neuronal relaxin receptor Lgr3 to couple growth to developmental timing. Nat. Commun. 6, 8732.

Honti, V., Cinege, G., Csordás, G., Kurucz, É., Zsámboki, J., Evans, C.J., Banerjee, U., and Andó, I. (2013). Variation of NimC1 expression in Drosophila stocks and transgenic strains. Fly (Austin). 7, 263268.

Hull-Thompson, J., Muffat, J., Sanchez, D., Walker, D.W., Benzer, S., Ganfornina, M.D., and Jasper, H. (2009). Control of Metabolic Homeostasis by Stress Signaling Is Mediated by the Lipocalin NLaz. PLoS Genet. 5, e1000460.

Martin-Blanco, E., Gampel, A., Ring, J., Virdee, K., Kirov, N., Tolkovsky, A.M., and Martinez-Arias, A. (1998). puckered encodes a phosphatase that mediates a feedback loop regulating JNK activity during dorsal closure in Drosophila. Genes Dev 12, 557-570.

McBrayer, Z., Ono, H., Shimell, M., Parvy, J.-P., Beckstead, R.B., Warren, J.T., Thummel, C.S., Dauphin- 
Villemant, C., Gilbert, L.I., and O'Connor, M.B. (2007). Prothoracicotropic hormone regulates developmental timing and body size in Drosophila. Dev. Cell 13, 857-871.

Milan, M., Campuzano, S., Garcia-Bellido, A., Milán, M., Campuzano, S., and García-Bellido, A. (1996). Cell cycling and patterned cell proliferation in the wing primordium of Drosophila. Proc Natl Acad Sci $U$ S A 93, 640-645.

Moskalev, A., Proshkina, E., Zhavoronkov, A., and Shaposhnikov, M. (2019). Effects of unpaired 1 gene overexpression on the lifespan of Drosophila melanogaster. BMC Syst. Biol. 13, 16.

Muzzopappa, M., Murcia, L., and Milán, M. (2017). Feedback amplification loop drives malignant growth in epithelial tissues. Proc. Natl. Acad. Sci. 114, E7291-E7300.

Osman, D., Buchon, N., Chakrabarti, S., Huang, Y.-T., Su, W.-C., Poidevin, M., Tsai, Y.-C., and Lemaitre, B. (2012). Autocrine and paracrine unpaired signaling regulate intestinal stem cell maintenance and division. J. Cell Sci. 125, 5944-5949.

Pasco, M.Y., and Léopold, P. (2012). High Sugar-Induced Insulin Resistance in Drosophila Relies on the Lipocalin Neural Lazarillo. PLoS One 7, e36583.

Pastor-Pareja, J.C., and Xu, T. (2013). Dissecting social cell biology and tumors using Drosophila genetics. Annu Rev Genet 47, 51-74.

Ponton, F., Chapuis, M.-P., Pernice, M., Sword, G.A., and Simpson, S.J. (2011). Evaluation of potential reference genes for reverse transcription-qPCR studies of physiological responses in Drosophila melanogaster. J. Insect Physiol. 57, 840-850.

Sisk, C.L., and Foster, D.L. (2004). The neural basis of puberty and adolescence. Nat. Neurosci. 7, 1040-1047.

Sotillos, S., Diaz-Meco, M.T., Moscat, J., and Castelli-Gair Hombria, J. (2008). Polarized subcellular localization of Jak/STAT components is required for efficient signaling. Curr Biol 18, 624-629.

Tennessen, J.M., and Thummel, C.S. (2011). Coordinating growth and maturation - insights from Drosophila. Curr Biol 21, R750-7.

Vallejo, D.M., Juarez-Carreño, S., Bolivar, J., Morante, J., and Dominguez, M. (2015). A brain circuit that synchronizes growth and maturation revealed through Dilp8 binding to Lgr3. Science 350, aac6767.

Wang, L., Sexton, T.R., Venard, C., Giedt, M., Guo, Q., Chen, Q., and Harrison, D.A. (2014). Pleiotropy of the Drosophila JAK pathway cytokine Unpaired 3 in development and aging. Dev. Biol. 395, 218-231. Yamanaka, N., Romero, N.M., Martin, F.A., Rewitz, K.F., Sun, M., O’Connor, M.B., and Léopold, P. 
(2013). Neuroendocrine Control of Drosophila Larval Light Preference. Science. 341, 1113-1116. 